\title{
Blood Components Involving Acceleration of Erythrocyte Sedimentation Rate, Particularly Red Cell Shape Factors
}

\author{
Akira FUROTA
}

\begin{abstract}
Summary Erythrocyte Sedimentation Rate testing (ESR) has long been used as a chronic inflammation marker. We evaluated the clinical usefulness of ESR with 20,926 laboratory testing data obtained from a multiphasic health testing system. 1) ESR data were studied with 49 items of clinical chemistry, hematology and physiology. Only globulin and hemoglobin exhibited significant correlation $(p>0.01)$. 2) ESR exhibited a negative correlation with MCV, but HGB did not. 3) The estimated multiple regressive equation was $\mathrm{Y}=-2.2546 \times$ hemoglobin value $(\mathrm{g} / \mathrm{d} l)+$ $9.6238 \times$ globulin value $(\mathrm{g} / \mathrm{d} l)+15.7124$. 4) Globulin increased with age but hemoglobin did not clearly decrease with it. 5) When the health testing data were applied to the estimation formula, $168 / 20,926(0.80 \%)$ were out of the $-11 \mathrm{~mm}$ area of the ESR regression line, and their showed hypochromic and microcytic in many cases. The results indicated that decreased hemoglobin means accelerated ESR but the anemia cases showing abnormally-low shape factors in $\mathrm{MCV}$ and $\mathrm{MCH}$ did not fit this assumption.
\end{abstract}

Key Words : ESR, Cell Shape, Anemia, Multi-variant Analysis

\section{Introduction}

ESR has been used for over 80 years throughout the world as a chronic inflammation marker ${ }^{1)}$. Testing method of ESR has been early established and standardized internationally and ESR is used widely because of its technical simplicity and low cost. Some physicians, however, say that ESR is old-fashioned and clinically less significant and is inferior to $\mathrm{CRP}$ as an inflammation marker. There are several reasons for this: (1)a non-specific inflammation marker, ( 2 ) inaccuracy of test-

Osaka City University Medical School Medical School, Department of Clinical and Laboratory Medicine ing, ( 3 ) large blood requirement $(1.6 \mathrm{~m} l$ whole blood), and ( 4 ) time consumption due to manual processing. Such a internationally stereotype concept for ESR is based on out-of-date laboratory data.

The multiphasic health testing (MHT) system has been popular by implementing a modern health check-ups by means of modern laboratory technology. In MHT circumstances the subjects of testing are mostly healthy company workers who pass active daily life. The outcome of testing helps examinees keep healthy conditions, to prevent development of life-style related diseases. All the tests in the system can be finished in a short period of time spending a minimum amount of blood, and the system 
provides many reliable test data with high accuracy and precision. The results of X-ray examination of chest and upper gastrointestinal tract, respiratory function tests, electrocardiography, fecal occult blood tests and serum tumor marker measurements are all simultaneously carried out within a short period time. Comprehensive diagnoses based on all test results, dietary consultation and life style education are conducted by a registered physicians licensed by the Japan Society of Multiphasic Health Testing within several hours following the test processes. Testing charge is generally considered reasonable. Thus, the MHT system has many merits for examinees: (1) fast and reliable testing processes, (2) acquisition of trends of yearly changes of the test results, ( 3 ) prevention and early detection of age ${ }^{-}$and life -style-related diseases, and ( 4 ) opportunity of early stage cancer detection. In the MHT system, usually either ESR or CRP is measured to detect the latent chronic inflammatory changes. To evaluate the efficacy of ESR on the modern laboratory technology, factors involved in acceleration of ESR were studied by analyzing the data obtained at MHT environment.

\section{Materials and Methods}

1. Blood samples were obtained from examinees who had multiphasic health testing at the Midori Health Care Foundation (Osaka).

\section{Test parameters}

Chemistry, physiological and other test items are listed in Table 1.

\section{Instruments}

Chemistry tests were conducted using a fullyautomated Hitachi 716 chemistry analyzer (Hitachi, Tokyo), and hematology tests were conducted with Coulter Counter Model-S (Coulter, Hialeah FL) of the system. All instruments were inspected thoroughly prior to
Table 1 Items examined by our multiphasic health testing system and means and SD values obtained

\begin{tabular}{|c|c|c|c|c|c|}
\hline \multirow{2}{*}{ No } & \multirow{2}{*}{ Item } & \multicolumn{2}{|c|}{ Male } & \multicolumn{2}{|c|}{ Female } \\
\hline & & Mean & SD & Mean & SD \\
\hline 1 & Gender & & & & \\
\hline 2 & Age & 49.15 & 14.45 & 47.11 & 13.20 \\
\hline 3 & Systolic Blood Pressure & 123.43 & 14.89 & 117.53 & 15.41 \\
\hline 4 & Diastolic Blood Pressure & 75.30 & 10.73 & 72.43 & 10.25 \\
\hline 5 & Intra Ocular Pressure (R) & 9.80 & 2.78 & 9.36 & 2.61 \\
\hline 6 & Optic Fund. Appearance (KW) & 0.26 & 0.45 & 0.18 & 0.38 \\
\hline 7 & Visual Power (R) & 0.47 & 0.46 & 0.56 & 0.45 \\
\hline 8 & Forced Vital Capacity & 530.68 & 93.07 & 352.10 & 60.04 \\
\hline 9 & Forced Expiratory Volume 1sec. & 305.92 & 69.60 & 229.82 & 48.33 \\
\hline 10 & Peak Flow & 384.63 & 72.81 & 278.19 & 51.20 \\
\hline 11 & Height & 167.11 & 6.16 & 154.54 & 5.31 \\
\hline 12 & Weight & 63.24 & 7.88 & 51.40 & 6.11 \\
\hline 13 & Body Mass Index & 119.97 & 12.70 & 100.99 & 11.58 \\
\hline 14 & Cardiac Length & 81.83 & 5.06 & 71.70 & 4.01 \\
\hline 15 & Thoracic Length & 38.39 & 3.81 & 33.93 & 3.76 \\
\hline 16 & Cardio Thoracic Ratio & 46.99 & 4.52 & 47.44 & 5.73 \\
\hline 17 & Heart Rate & 61.92 & 8.54 & 66.77 & 9.34 \\
\hline 18 & Power of Hearing $(\mathrm{R} 8000 \mathrm{~Hz})$ & 2.41 & 1.71 & 1.64 & 0.94 \\
\hline 19 & PSP Test & 33.16 & 5.62 & 35.25 & 6.26 \\
\hline 20 & Erythrocyte Sedimentation Rate & 6.46 & 5.02 & 11.26 & 6.55 \\
\hline 21 & $\mathrm{zTT}$ & 6.95 & 2.50 & 7.98 & 2.50 \\
\hline 22 & LDH & 239.35 & 35.72 & 251.98 & 42.53 \\
\hline 23 & GPT & 12.78 & 5.60 & 9.34 & 3.27 \\
\hline 24 & GOT & 16.38 & 3.78 & 14.95 & 3.23 \\
\hline 25 & Alkaline Phosphatase & 5.69 & 1.40 & 5.32 & 1.67 \\
\hline 26 & Uric Acid & 5.69 & 1.11 & 4.17 & 0.81 \\
\hline 27 & Cholesterol & 188.38 & 29.85 & 194.46 & 34.40 \\
\hline 28 & Total Protein & 6.98 & 0.35 & 7.04 & 0.33 \\
\hline 29 & Albumin & 4.54 & 0.23 & 4.51 & 0.20 \\
\hline 30 & Globulin & 2.45 & 0.26 & 2.53 & 0.25 \\
\hline 31 & $A / G$ & 1.87 & 0.22 & 1.80 & 0.19 \\
\hline 32 & Blood Urea Nitrogen & 14.90 & 2.85 & 14.00 & 2.91 \\
\hline 33 & Total Bilirubin & 0.80 & 0.25 & 0.68 & 0.22 \\
\hline 34 & Triglyceride & 101.39 & 45.10 & 73.91 & 30.64 \\
\hline 35 & Creatinine & 1.25 & 0.14 & 0.99 & 0.12 \\
\hline 36 & Acid Phosphatase & 1.98 & 0.27 & 1.75 & 0.30 \\
\hline 37 & $\gamma-\mathrm{GTP}$ & 20.22 & 14.70 & 8.80 & 4.77 \\
\hline 38 & $\mathrm{Na}$ & 142.69 & 1.67 & 142.34 & 1.86 \\
\hline 39 & $\mathrm{~K}$ & 4.29 & 0.26 & 4.22 & 0.30 \\
\hline 40 & Fasting Blood Glucose & 98.94 & 6.63 & 93.91 & 5.77 \\
\hline 41 & Glucose Tolerance Test (1hr.) & 144.26 & 27.36 & 142.25 & 29.34 \\
\hline 42 & Hemoglobin $A_{1} C$ & 5.11 & 0.41 & 4.95 & 0.35 \\
\hline 43 & $\mathrm{RBC}$ & 464.08 & 32.99 & 419.37 & 25.81 \\
\hline 44 & WBC & 69.77 & 15.24 & 62.31 & 12.71 \\
\hline 45 & MCV & 93.63 & 4.03 & 90.61 & 4.17 \\
\hline 46 & Hemoglobin & 14.81 & 0.94 & 12.77 & 0.85 \\
\hline 47 & $\mathrm{MCH}$ & 31.95 & 1.39 & 30.49 & 1.62 \\
\hline 48 & $\mathrm{MCHC}$ & 34.13 & 0.75 & 33.64 & 0.81 \\
\hline 49 & Hematocrit & 43.38 & 2.49 & 37.96 & 2.27 \\
\hline
\end{tabular}

daily-routine services and always monitored with computerized internal quality control programs $^{2,3)}$.

All data were stored in a large-scale computer to create databases.

\section{ESR}

The test was performed at the room temperature by the standard method proposed by the International Council for Standardization in Hematology $(\mathrm{ICSH})^{4)}$.

ICSH defines to determine only 60 minute value instead of 120 minute value, because the latter was judged to have less clinical significance. 


\section{Statistic analysis}

To void misjudgment due to interference caused by too strong correlation that may happen when plural test items of similar nature with such as hemoglobin and hematocrit are both applied as the objects of partial correlation analysis, we used either one of them as an object of our study ${ }^{5,6}$.

Therefore the parameters are grouped in related items and used one of them for partial correlation analysis.

Including multi-regression analysis and correlation analysis were conducted using StatView computing software (Microsoft).

\section{Results}

\section{Chemical test profile obtained by MHT system}

Table 1 shows the means and standard deviations for each parameter studied.

Fig. 1 shows the ESR distribution and changes by gender and age. ESR values exhibited a log normal distribution and aged population showed higher ESR values. Female showed basically accelerated ESR than male. Changes due to age were also slightly stronger in females than in males.

Simple correlation and partial correlation analysis revealed that globulin and hemoglobin were significantly correlated with ESR changes (Table 2).

Age, retinal photography, spirometry, audiometry, PSP, globulin and hemoglobin are correlated with ESR in simple correlation. Their parameter values are related with aging trend. Partial correlation analysis was conducted, and hemoglobin and globulin showed higher $r$-values with ESR. Based on these results, we confirm the aging trend for hemoglobin, globulin and $\mathrm{A} / \mathrm{G}$ ratio and $\mathrm{ESR}$ correlation with globulin, hemoglobin in each individual Fig. 2, Fig. 3.

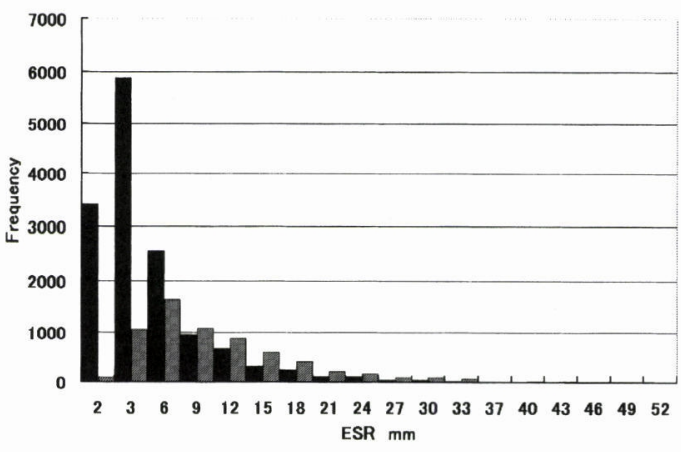

(a)

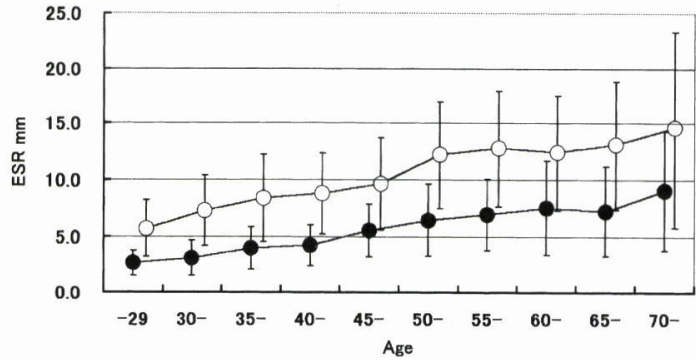

(b)

Fig. 1 ESR histograms and age changes

(a) Histogram of ESR in males (ם) and females ( $\square$ )

(b) ESR changes by age in males ( and females $(\mathrm{O})$

Table 2 Results of simple and partial correlation analysis

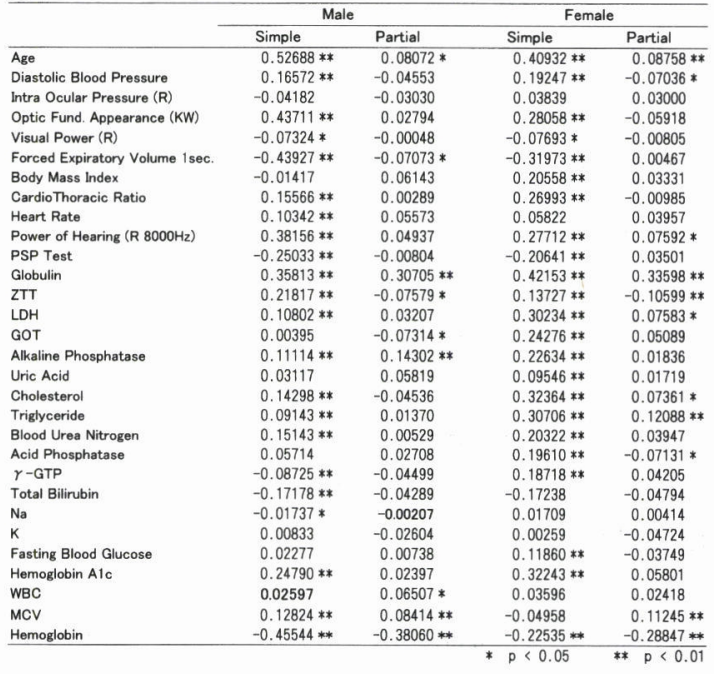

2. Multiple regression analysis for globulin and hemoglobin

To examine the relationships among ESR, 


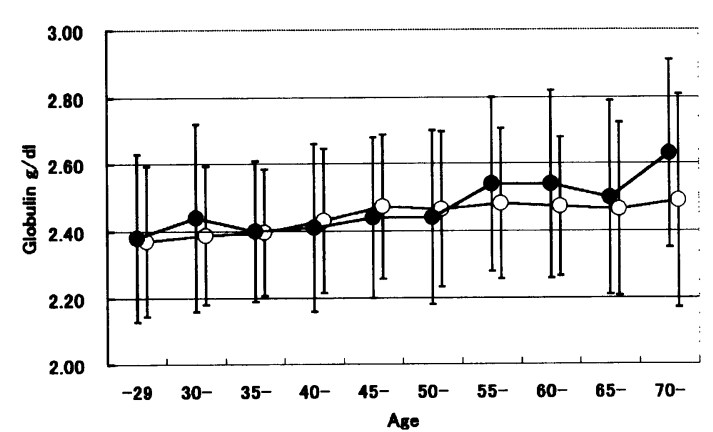

(a)

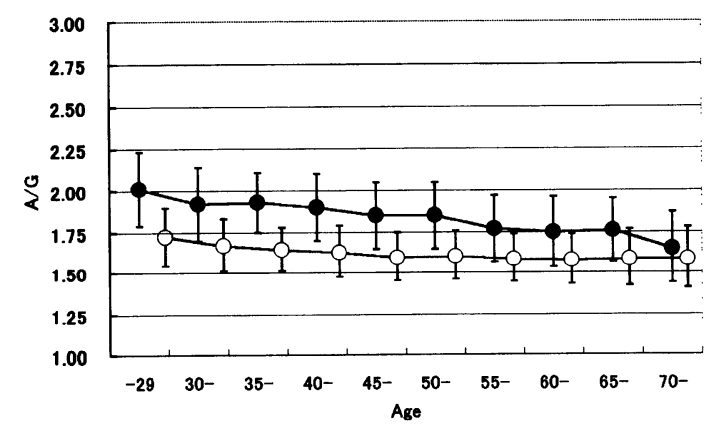

(b)

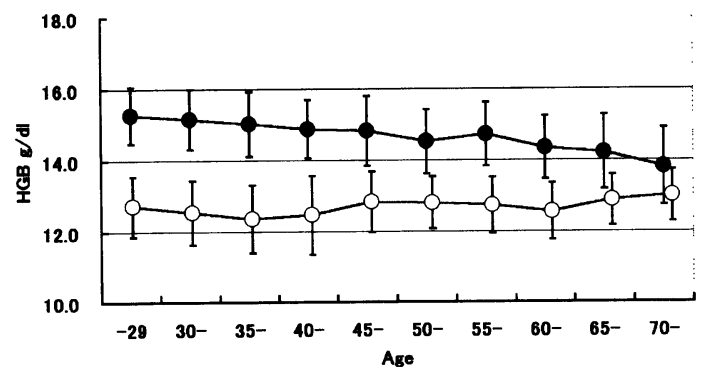

(c)

Fig. 2 Relations with aging among globulin (a), albumin globulin ratio (b), and hemoglobin (c) in males $(\mathbf{O})$ and females $(O)$

globulin and hemoglobin, multiple regression analysis was implemented using globulin and hemoglobin values. The equation of $\mathrm{Y}=$ $-2.2546 \times$ hemoglobin value $(\mathrm{g} / \mathrm{d} l)+9.6238 \times$ globulin value $(\mathrm{g} / \mathrm{d} l)+15.7124$ was obtained.

3. Fitness of the globulin-hemoglobin-ESR (GHE) equation to actually determined values

Fig. 4 shows scatter plots of residues between actually determined values and predicted ESR values obtained by the GHE equation. Fig. 5

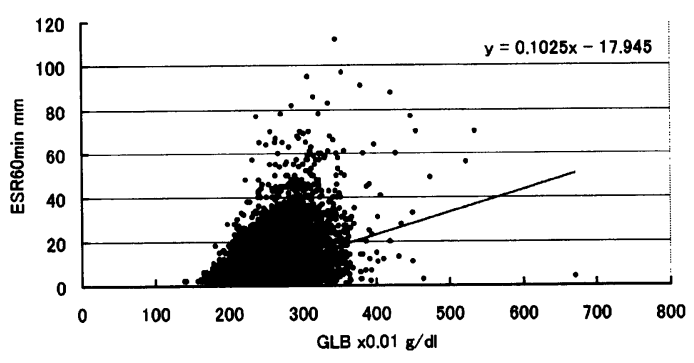

(a)

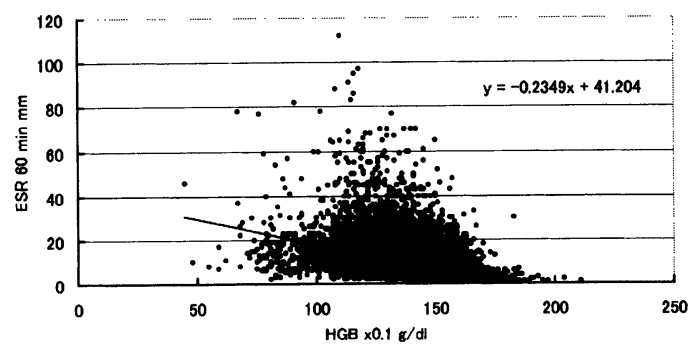

(b)

Fig. 3 Scatter plots of ESR between globulin (a), hemoglobin ( $b$ )

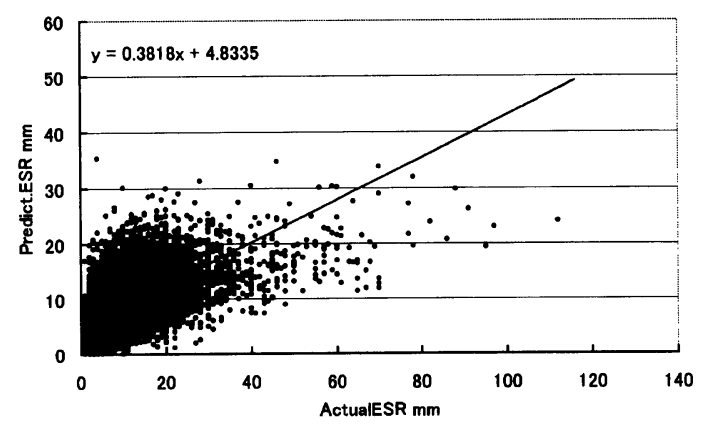

Fig. 4 Scatter plots of residues between actual determined value and predicted ESR value obtained by the equation

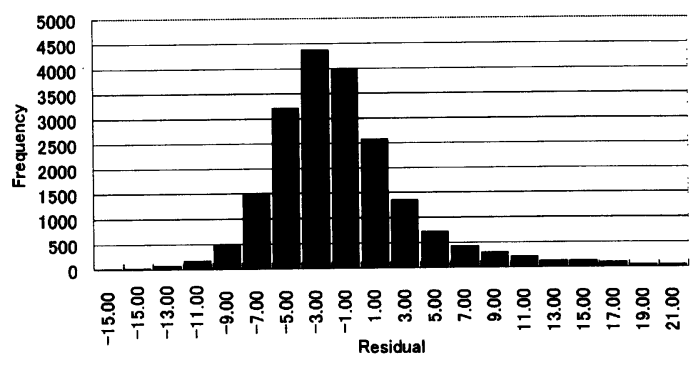

Fig. 5 Distribution curve of residues (differences) between actually determined values and predicted values 
Table 3 Cross table for residues (differences) between actual determined values and predicted values. $\mathrm{X}$ axis : actual values, $\mathrm{Y}$ axis : predicted values

\begin{tabular}{|c|c|c|c|c|c|c|c|c|c|c|c|c|c|c|c|}
\hline & 3 & 4. & 8. & 12. & 16. & 20. & 24. & 28. & 32. & 36. & 40. & 44. & 48. & 52. & Total \\
\hline 3 & 3,208 & 856 & 127 & 45 & 14 & 6 & 1 & 1 & 0 & 0 & 0 & 0 & 0 & 0 & 4,258 \\
\hline 4. & 2,914 & 3.008 & 880 & 330 & 109 & 49 & 23 & 11 & 3 & 1 & 3 & 0 & 0 & 0 & 7,331 \\
\hline 8. & 577 & 2,249 & 1,464 & 774 & 326 & 162 & 90 & 43 & 19 & 5 & 10 & 4 & 2 & 5 & 5,730 \\
\hline 12. & 56 & 425 & 704 & 586 & 331 & 229 & 136 & 81 & 53 & 18 & 15 & 12 & 6 & 12 & 2.664 \\
\hline 16. & 8 & 57 & 106 & 132 & 113 & 99 & 57 & 32 & 25 & 12 & 10 & 13 & 9 & 21 & 694 \\
\hline 20. & 2 & 8 & 25 & 36 & 31 & 27 & 12 & 9 & 10 & 4 & 4 & 2 & 2 & 16 & 188 \\
\hline 24. & 0 & 1 & 2 & 7 & 6 & 6 & 5 & 6 & 1 & 1 & 1 & 3 & 1 & 5 & 45 \\
\hline 28. & 0 & 0 & 1 & 0 & 2 & 2 & 0 & 1 & 0 & 0 & 1 & 0 & 0 & 6 & 13 \\
\hline 32. & 0 & 1 & 0 & 0 & 0 & 0 & 0 & 0 & 0 & 0 & 0 & 1 & 0 & 1 & 3 \\
\hline 36. & 0 & 0 & 0 & 0 & 0 & 0 & 0 & 0 & 0 & 0 & 0 & 0 & 0 & 0 & 0 \\
\hline 40. & 0 & 0 & 0 & 0 & 0 & 0 & 0 & 0 & 0 & 0 & 0 & 0 & 0 & 0 & 0 \\
\hline 44. & 0 & 0 & 0 & 0 & 0 & 0 & 0 & 0 & 0 & 0 & 0 & 0 & 0 & 0 & 0 \\
\hline 48. & 0 & 0 & 0 & 0 & 0 & 0 & 0 & 0 & 0 & 0 & 0 & 0 & 0 & 0 & 0 \\
\hline 52. & 0 & 0 & 0 & 0 & 0 & 0 & 0 & 0 & 0 & 0 & 0 & 0 & 0 & 0 & 0 \\
\hline Total & 6,765 & 6,605 & 3,309 & 1,910 & 932 & 580 & 324 & 184 & 111 & 41 & 44 & 35 & 20 & 66 & 20,926 \\
\hline
\end{tabular}

Table 4 Subgroups of residue distribution. Means and SD of globulin, A/G ratio, red cell count, $\mathrm{MCV}, \mathrm{MCH}$, and hemoglobin in each subgroup. Group 1 (less than $-11 \mathrm{~mm}$ ), group 2 (between -10 and $-6 \mathrm{~mm}$ ), group 3 (between -5 and $4 \mathrm{~mm}$ ), group 4 (between 5 and $9 \mathrm{~mm}$ ), group 5 (between 10 and $14 \mathrm{~mm}$ ), group 6 (greater than $15 \mathrm{~mm}$ )

\begin{tabular}{|c|c|c|c|c|c|c|c|c|c|c|c|c|}
\hline \multirow[b]{3}{*}{$G \perp B$} & \multicolumn{2}{|l|}{ I } & \multicolumn{2}{|l|}{ II } & \multicolumn{2}{|l|}{ III } & \multicolumn{2}{|c|}{ IV } & \multicolumn{2}{|l|}{$\mathrm{V}$} & \multicolumn{2}{|c|}{ VI } \\
\hline & Mean & SD & Mean & SD & Mean & SD & Mean & SD & Mean & SD & Mean & SD \\
\hline & 2.87 & 0.52 & 2.71 & 0.28 & 2.47 & 0.27 & 2.53 & 0.34 & 2.69 & 0.33 & 2.81 & 0.43 \\
\hline$A / G$ & 1.57 & 0.24 & 1.67 & 0.17 & 1.86 & 0.21 & 1.81 & 0.26 & 1.68 & 0.22 & 1.60 & 0.24 \\
\hline RBC & 428.41 & 40.12 & 441.58 & 37.02 & 457.31 & 39.50 & 441.15 & 40.39 & 432.20 & 38.32 & 422.44 & 41.43 \\
\hline MCV & 79.43 & 12.35 & 90.44 & 6.54 & 93.26 & 4.74 & 93.34 & 5.40 & 92.95 & 5.75 & 92.92 & 6.18 \\
\hline HGB & 10.96 & 2.57 & 13.40 & 1.58 & 14.45 & 1.38 & 13.92 & 1.53 & 13.52 & 1.36 & 13.18 & 1.53 \\
\hline $\mathrm{MCH}$ & 25.52 & 5.23 & 30.35 & 2.64 & 31.62 & 1.77 & 31.57 & 2.04 & 31.32 & 2.17 & 31.22 & 2.36 \\
\hline Numbers & 1760. & & $2,166(10$ & $35 \%)$ & $16,214(77$ & $.45 \%)$ & $1,366(6$ & $3 \%)$ & $504(2.4$ & & $500(2$. & \\
\hline
\end{tabular}

shows a distribution curve of residues (differences) between actual determined values and predicted values. The curve shows a normal distribution, with a median of about minus 3.00 .

Table 3, expressed with actual numbers, shows a cross table for the Fig. 4.

The residue group was subdivided into 6 groups by the residue power: group 1 (less than $-11 \mathrm{~mm}$ ), group 2 (between -10 and -6 $\mathrm{mm}$ ), group 3 (between -5 and $4 \mathrm{~mm}$ ), group 4 (between 5 and $9 \mathrm{~mm}$ ), group 5 (between 10 and $14 \mathrm{~mm}$ ), group 6 (greater than $15 \mathrm{~mm}$ ).

Table 4 shows mean and SD values for globulin, $\mathrm{A} / \mathrm{G}$ ratio, red cell count, $\mathrm{MCV}, \mathrm{MCH}$, and hemoglobin in each subgroup.

The results showed that group 1 had low MCV, hemoglobin and $\mathrm{MCH}$.

Fig. 6 shows the distribution curves for red cell count, hemoglobin, $\mathrm{MCV}$ and $\mathrm{MCH}$ in each subgroup. The curve patterns for red cell count were very similar for the 6 subgroups. However, distribution curves for hemoglobin $\mathrm{MCV}$ and $\mathrm{MCH}$ of group 1 were different from those in the other subgroups. These facts suggested that not only the volume factor but also the intracellular hemoglobin factor plays an important role in regulating acceleration of ESR.

\section{Discussion}

Recent laboratory technique provides accurate and precise data spending a minimum amount of blood. Rapid and steady health check-ups can be implemented by the modeled testing protocols, MHT system. However, what laboratory analyzers can produce are the data and it is not the health information. The information is produced by person who use 

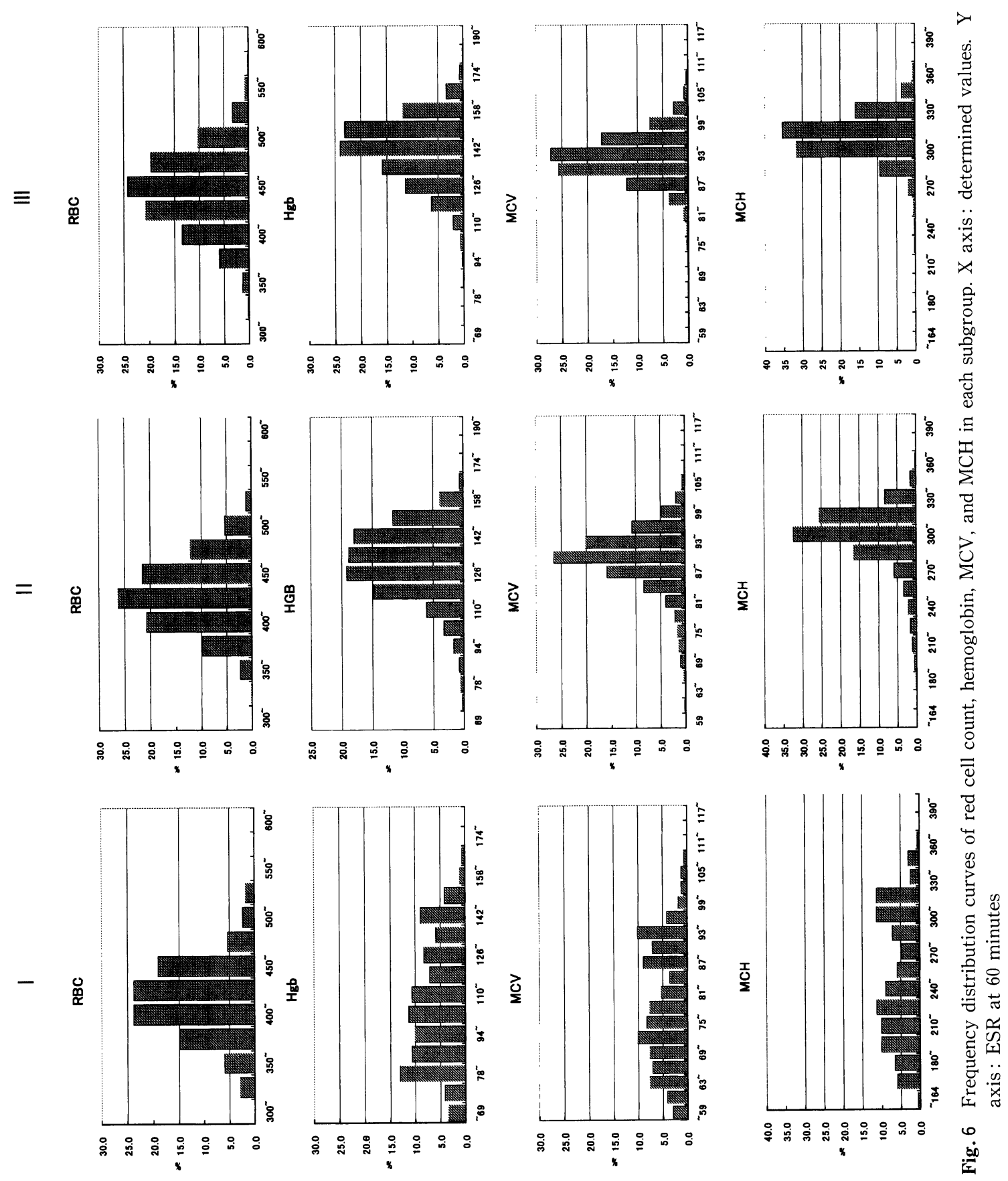

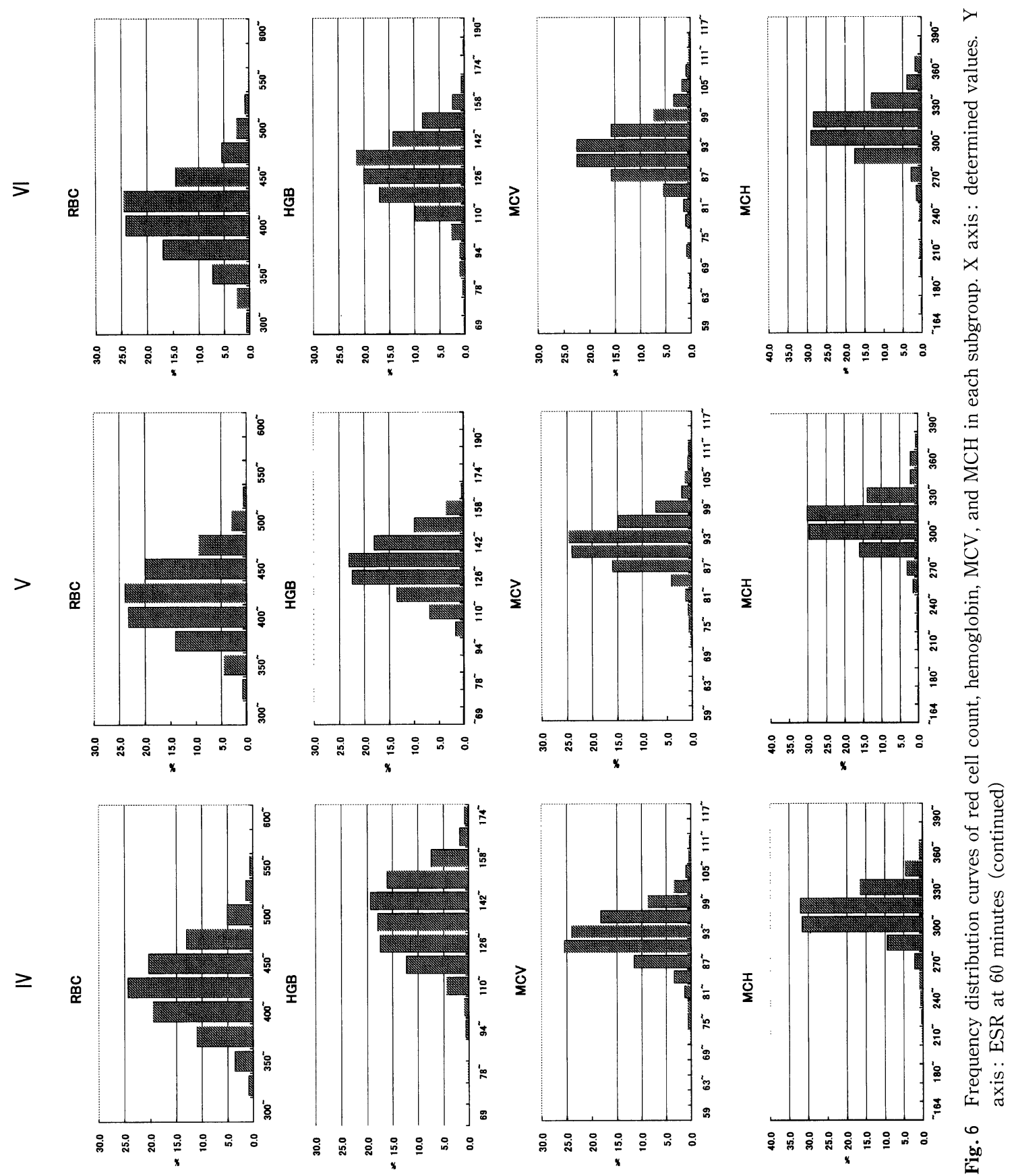
laboratory tests. The MHT system can be called a standardized diagnostic methodology because it usually has a well-organized qualification system in the facility or implement guaranteed external control system. In the system, ESR would play an important role for health monitoring because of its simplicity, inexpensiveness and reliablity. ESR is one of the most popular tests currently used all over the world. widely ${ }^{7 \sim 10}$.

ESR test usually takes 2 hour to perform and spends $2.0 \mathrm{~m} l$ citrated blood. Because of its technical simplicity and diagnostic value, many kinds of simplified ESR systems has been developed. However, the most reliable methods is Westergren's one which is commonly used in Japan.

Clinical usefulness of ESR is still controversial because of its non-specificity. The mechanism of CRP elevation is fundamentally different from that of ESR acceleration. CRP (half life: $5 \sim 7$ hours) is good as an acute inflammation marker reflecting an increase of reactants with short life such as fibrinogen (half life: $3.2 \sim 4.5$ days) in acute phase of inflammation, while ESR is accelerated by an increase of gamma globulin (half life : 23 days) associated with a decrease of albumin (half life : 19 days) in chronic inflammation ${ }^{11}$.

Fibrinogen is a major accelerating factor of ESR, but its measurement can not be conducted by a regular automated analyzing apparatus and requires a blood clotting method using an automated coagulometer. Its accuracy and precision are not yet satisfactory, so we excluded the discussion about fibrinogen in this study.

In the correlated items with ESR by simple correlation, simultaneously indicated correlation in partial correlation were hemoglobin, globulin.

The characteristics of test items significantly correlated to ESR in simple correlation study are all related to aging.

But the majority of their items did not correlate in partial correlation. This result suggests that the correlation exists in all ages.

In the relation with age among globulin, hemoglobin, globulin value increased in male and female, hemoglobin value in male decreased by aging and closely related female at 70, in female not changed all age Fig. $2(\mathrm{c})^{13)}$.

ESR values increased in males and females Fig. 1 (b). This finding suggests that globulin affects ESR more strongly than hemoglobin Fig. 2(b), (c).

Our partial correlation studies revealed that globulin affects ESR positively and hemoglobin affects it negatively. Globulin increases with age, although hemoglobin is not affected by aging. Accelerated ESR in the aged can be explained as an effect of globulin. However, the story of hemoglobin action is different form that of globulin.

Acceleration of ESR is observed when blood hemoglobin level is low.

However, hemoglobin itself is enclosed in the red cell and it has no direct activity of ESR acceleration. The ESR acceleration is only an outcome brought by hemoglobin containing red cell behavior.

ESR process is classified into 3 stages; 1 . Red cell rouleau aggregation, 2. fast-setting and 3 . packing periods. In the 1 st period, rouleaux formation occurs. Rapid but isospeed sedimentation occurs in the 2 nd period $^{14,15)}$.

Basically, ESR phenomenon is explained by the following Stokes's equation.

Gravitational force

$\mathrm{Fd}=\left(4 \mathrm{r}^{3} \pi / 3\right) \times\left(\rho_{1}-\rho_{2}\right) \times \mathrm{g}$

( $\mathrm{r}=$ sphere radius, $\rho_{1}=$ sphere density, $\rho_{2}=$ liquid density, $\mathrm{g}=$ gravity)

Viscous resisting force

$$
\mathrm{F}_{\mathrm{V}}=6 \pi \eta \mathrm{r}
$$


$(\eta=$ plasma viscocity $)$

sphere sedimentation velocity $(\mathrm{V})$ is expressed by the formula of

$$
\mathrm{V}=\mathrm{Fd} / \mathrm{Fv}_{\mathrm{V}}=(2 / 9) \times\left(\mathrm{r}^{2} / \eta\right) \times\left(\rho_{1}-\rho_{2}\right) \times \mathrm{g}
$$

$\mathrm{V}$ in this formula is called Stokes' $\mathrm{s}$ velocity $^{16 \sim 19)}$.

In the equation, hematocrit, rouleaux formation, size of rouleaux, red cell interaction, red cell membrane charge, plasma protein and serum viscosity are inflammatory factors of blood. However, involvement of hemoglobin was not mentioned. However, our data demonstrated that hemoglobin is one of major factors in red cells for acceleration of ESR.

Our study was designed to get simple correlation among laboratory data first and to get partial correlation among them. Red cell count, hematocrit, and hemoglobin showed similar tendencies to the partial correlation analysis, but hemoglobin gave us the highest $r$ values among the there, which indicating the importance of hemoglobin of the ESR acceleration.

A general tendency of more accelerated ESR of female than of male was interpreted caused by low red cell count, hematocrit and hemoglobin of female. With simple and partial correlation analysis, globulin and hemoglobin were extracted statistically as major factors accelerating ESR. Globulin is a age-dependent factor, but hemoglobin is not.

As the role of hemoglobin on ESR acceleration was not clear, we tried to study its cause by means of statistic analysis. Hemoglobin was a less age-relating issue in our subjects. To examine the relationships among ESR, globulin and hemoglobin, multiple regression analysis was implemented using globulin and hemoglobin values. The equation $\mathrm{Y}=-2.2546 \times$ hemoglobin value $(\mathrm{g} / \mathrm{d} l)+9.6238 \times$ globulin value $(\mathrm{g} / \mathrm{d} l)+15.7124$ was obtained. The fitness of the globulin-hemoglobin-ESR (GHE) equation to actually determined values was tested, and scatter plots of residues on the GHE equation exhibited a normal distribution. Its median positioned to around minus 3.00.

Residues between actual ESR value and predicted value showed normal distribution curve, and its peak shifted to the negative side, so-called to the accelerated side. The residue group was subdivided into 6 groups, and relationship between residues and red cell parameters were examined. Its results showed that group I, hypochromic and microcytic groups showed high difference in the residues. That results indicates that red cells with low hemoglobin content and small in size showed not so high ESR. The reason that we did not evaluate ESR in comparison with $\mathrm{MCHC}$ is that electric MCHC was described to be not good as an anemia indicator ${ }^{20)}$.

Anemia is due to either low hemoglobin or low red cell count. WHO anemia classification defines to diagnose anemia with hemoglobin or hematocrit, because both changes in parallel. However, anemia can be classified into various types by its cell, volume and cell hemoglobin content. Previous concept defined that, anemia shows accelerated $\mathrm{ESR}^{8,21}$.

However, our results supported by large numbers of data indicated that anemia is not sure to show accelerated ESR. Anemias with low MCV and MCH did not show highly accelerated ESR. Probably specific cell shape and size involving MCV and MCH would be responsible for non-retarded ESR in anemia.

$\mathrm{HGB}, \mathrm{MCV}, \mathrm{MCH}$ histogram of group 1 which is subdivided residue show plateau pattern to other groups. The results suggest existence of plural characteristic population for example aniso_cytes (size) or poikilo_cytes (shape). This mean though monocytic erythrocyte is easily to make rouleaux formation but abnormal size or shape cytes is not ${ }^{2224)}$.

Population increase is a worldwide concern 
in the late 20 th century. On the other hand, developed countries suffer from the decrease of total population with partial increase of aged population, and the life style relating diseases (LSRD) become one of critical social problems in those countries.

To keep good health and prevent LSRD, Multiphasic Health Check-up Testing Systems were developed, which contribute health promotion of healthy workers. In the system, various types of testing are performed with fullyautomated modern instruments, and many reliable test data are provided accurately and precisely. Erythrocyte Sedimentation Rate testing (ESR) is one of such examinations.

\section{Acknowledgement}

I thank Prof. Dr. Noriyuki TATSUMI for his guidance to accomplish this study. I worked as a staff of The Midori Heath Care Foundation and I appreciate all efforts of my colleague serving there.

\section{References}

1) Westergren, A., Studies of the sedimentation stability of the blood in pulmonary tuberculosis, Acta Med. Scandi., 54, 247-287, 1921.

2) Furota, Akira, Miyagawa, Teikichi, Tsuda, Izumi, Tatsumi, Noriyuki: Algorithm for Detection of Accidental Errors with Automated Blood Cell Counting Using Regression Analysis. The $6^{\text {th }}$ International Symposium on Quality Control-Osaka. 399-407, Excepta Medica, Ltd, Tokyo, 1988.

3) Tatsumi, Noriyuki, Tsuda, Izumi, Furota, Akira : Use of Control Materials in the Hematology Laboratory. The $6^{\text {th }}$ International Symposium on Quality Control-Osaka, 129-142, Excepta Medica, Ltd, Tokyo, 1988.

4) Members of expert panel, ICSH recommendations for measurement of erythrocyte sedimentation rate, J. Clin. Pathol., 46, 198-203, 1993.

5) Ryuichi Kobayashi, Simple correlation and partial correlation, in Introduction to Correlation, Regression Analysis, 208-209, Nikkagiren, Tokyo, 1987.

6) Shoji Goto, ChoichiroTomonaga, Interpretation of correlation coefficients, Multi Variant Data Analysis, 16-18, Kagakujyohosha, Osaka, 1975.

7) Glonlie M, Hjortdahl P, The erythrocyte sedimentation rate: its use and usefulness in primary health care., Scand. J. Prim. Health Care, 9, 87-102, 1991.

8) K. Fukutake, T. Mikami, 18. Erythrocyte Sedimentation Rate, Specific gravity and viscosity of Blood, NipponKetsuekigakuzusho 13, 573-611, Maruzen, Tokyo, 1979.

9) Kumasaka, K, Kubo, N., Inflammatory reaction and laboratory tests : hematologic examination, Rinsho Byori, 38, 260-266, 1990.

10) Saadeh $C$. ., The erythrocyte sedimentation rate: old and new clinical applications., South Med. J., 91, 220-225, 1998.

11) Kinetics of metabolism in component of Blood, Editor: JapanBioChemicalSociety, BioChemicalDataBook (2), 640-641, Tokyo Chemical Coterie, Tokyo, 1982.

12) Maeda, N., Seike, M., Shiga, T., Erythrocyte aggregation induced by fibrinogen_Interaction between fibrinogen and erythrocytes, J. Jpn. Soc. Biorheol., 1, 27-37, 1987.

13) Tsuda, I., Furota, A., Miyagawa, T., Tatsumi, N., Reference values for hematological tests in multiple health testing systems, JMHTS, 15, 233-238, 1989.

14) Thomas L. Fabry, Mechanism of Erythrocyte Aggregation and Sedimentation, Blood, 70, 15721576, 1987.

15) D. R. Forsdyke, P. M. Ford, Rouleaux formation as a measure of the phase separating ability of plasama, J. Theor. Biol., 103, 467-472, 1983.

16) Shoten Oka, A note on a theoretical study of erythrocyte sedimentation, Biorheology, 20, 579-581, 1983.

17) Shoten Oka, A Physical Theory of Erythrocyte Sedimentation, Biorheology, 22, 315-321, 1985.

18) J. Mayer, Z. Pospisil, J. Litzman, The Mechanism of Erythrocyte Sedimentation in Westergren' $\mathrm{s}$ Examonation, Biorheology, 29, 261-271, 1992.

19) V. G. Petrov, I. Edissonov, The role of aggregation kinetics in the sedimentation of erythrocytes, Biorheology, 33, 353-364, 1996.

20) Miwa, Shiro: Erythrocyte sedimentation rate, Editor: Miwa Shiro, In Hematology Vol. 3. Modern Medical Technology, 112-115, Igaku Shoin Ltd. Tokyo, 1972.

21) Kanfer EJ, Nicol BA., Haemoglobin concentration and erythrocyte sedimentation rate in primary care patients, J R Soc. Med., 90, 16-8, 1997.

22) Walter H. Reinhart, Ajit Sing, P. Werner Straub, Red Blood Cell aggregation and sedimentation: the role of the cell shape, British Journal of Haematology, 1989, 73, 551-556.

23) Christine Lawrence, Mary E. Fabry, Erythrocyte Sedimentation Rate During State and Painful Crisis in Sickle Cell Anemia, The American Journal of Medicine, 81, 801-808, 1986. 
Blood Components Involving Acceleration of Erythrocyte Sedimentation Rate, Particularly Red Cell Shape Factors

24) A. Singth, W. H. Reinhart, The influence of fraction of abnormal erythrocyte on aggregation, European
Journal of Clinical Investigation, 21, 597-600, 1991. 